\title{
The Use of Direct Current Stimulation to Investigate the Role of Each Hemisphere in Motor Learning of Reaching Task
}

\author{
Mostafa Teymuri Kheravi ${ }^{1}$, Alireza Saberi Kakhki ${ }^{1 *}$, Hamidreza Taheri ${ }^{1}$, Ali Ghanaie Chaman Abad ${ }^{2}$, Mohammad \\ Darainy $^{3}$
}
${ }^{1}$ Department of Motor Behavior, Ferdowsi University of Mashhad, Mashhad, Iran
${ }^{2}$ Department of Psychology, Ferdowsi University of Mashhad, Mashhad, Iran
${ }^{3}$ Department of Psychology, McGill University, Montreal, Canada

\section{ABSTRACT}

Introduction: Transcranial Direct Current Stimulation (tDCS) can improve or impair the function of the brain. This has turned tDCS into a tool that can be used for evaluation of hemispheric specialization in motor programming and final position accuracy, as components of motor control and learning. Materials and Methods: Two different studies were designed. 53 male students ( $21.34 \pm 1.61$ years) and 43 male students ( $20.442 \pm 1.578$ years) were participated in the first and second studies, respectively. Participants were randomly assigned into four groups. $\mathrm{C} 3 / \mathrm{C} 4$ and F3/F4 areas were stimulated with the $2 \mathrm{~mA}$ current in the first and second studies, respectively. The Repeated Measure test was used to analyze data. Results: In the first experiment, left M1 group (left anode/right cathode stimulation) significantly improved motor programming compared to the other groups. In the second experiment, the right dorsolateral prefrontal cortex group (right cathode/left anode) significantly decreased final position accuracy compared to the other groups. Conclusion: Our data suggested that the left hemisphere is specialized for motor programming whereas the right hemisphere is specialized for final position accuracy. These results are interpretable with hybrid motor control hypothesis.

Key words:

1. Brain

2. Learning

3. Role

*Corresponding Author: Alireza Saberi Kakhki

E-mail: askakhki@um.ac.ir 


\section{استفاده از تحريك جريان مستقيم جهت بررسى نقش هر يك از نيمكرههاى مغز در يادكيرى و كنترل

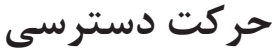

مصطفى تيمورى خروى'، عليرضا صابرى كاخكى'"، حميدرضا طاهرى'، على غنايى خمن آباد'، محمد دارينى"

$$
\begin{aligned}
& \text { 'كروه رفتار حركتى، دانشگاه فردوسى مشهد، مشهه، ايران } \\
& \text { "كروه روانشناسى، دانشكاه فردوسى مشهر، مشهد، ايران } \\
& \text { "كروه روانشناسى، دانشحاه مخيل، مونترال، كانادا }
\end{aligned}
$$

\section{اطلاعات مقاله: - إن}

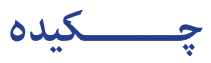

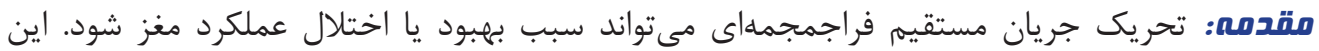

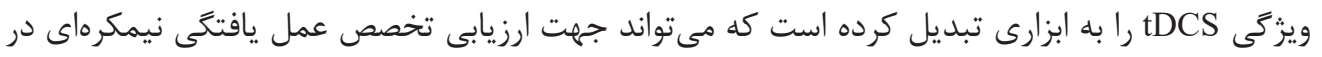

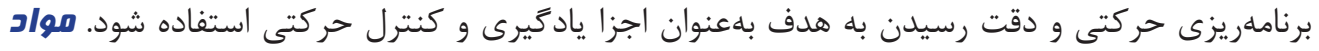

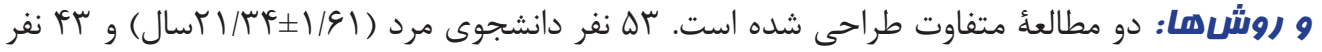

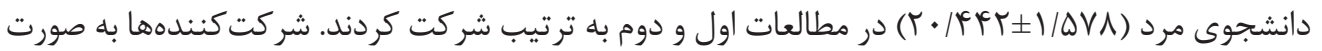

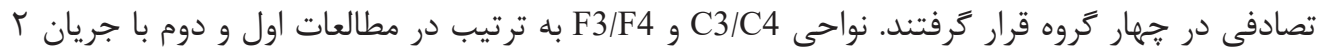

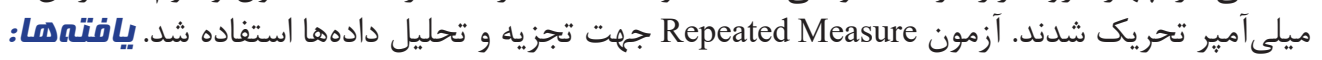

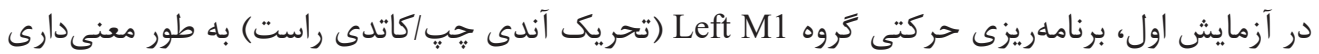

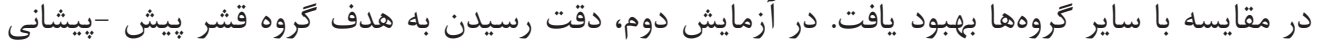

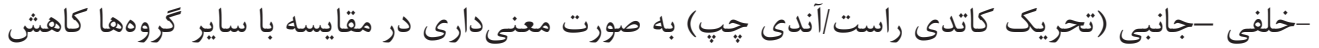

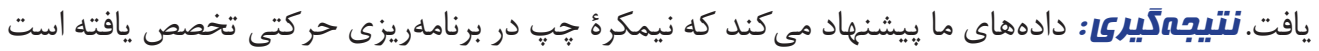

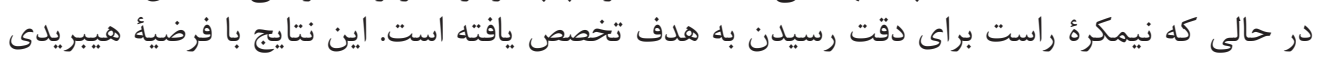
كنترل حركت قابل تفسير است.

كليد وازهها:

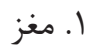

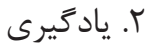
بق 


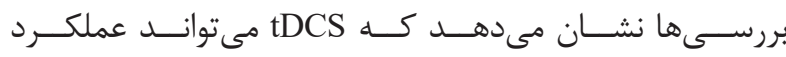

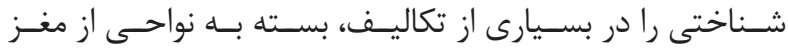

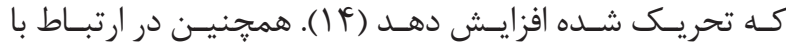

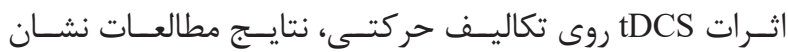

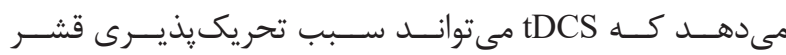

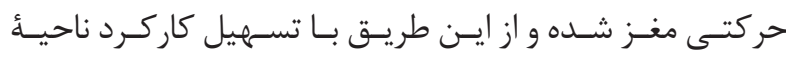

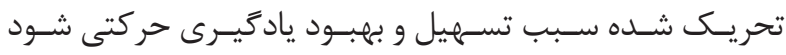

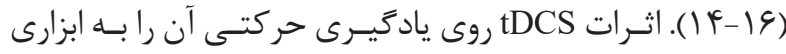

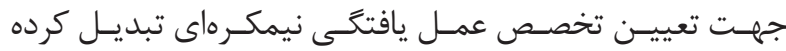

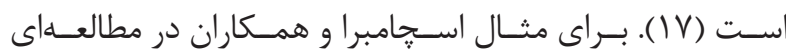

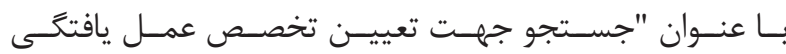

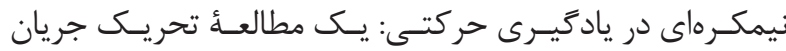

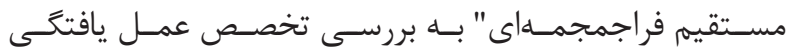

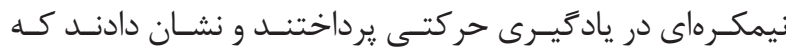

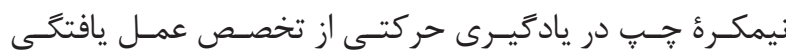

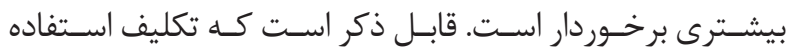

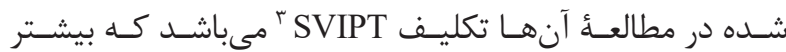

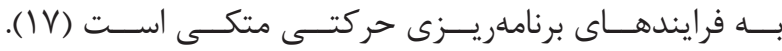

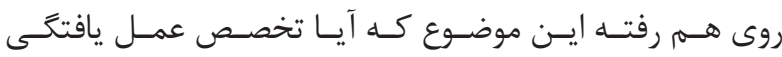

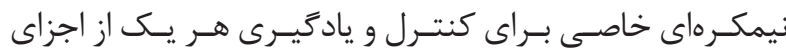

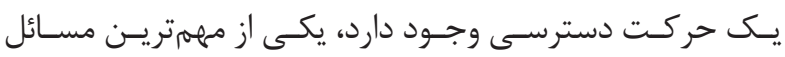

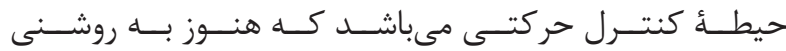

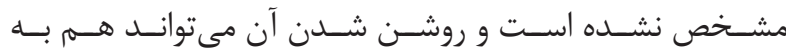

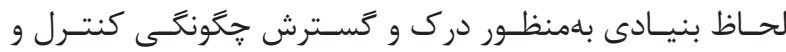

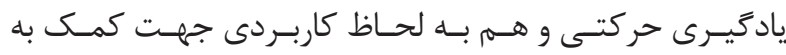

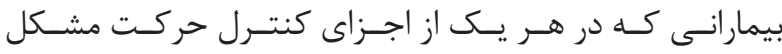

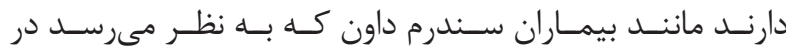

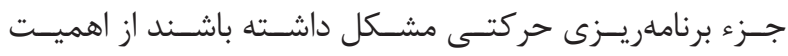
زيـادى برخــوردار باشــد.

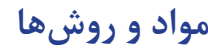
آزمايش اول

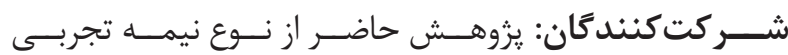

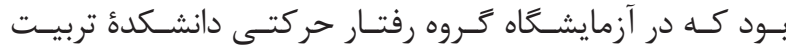

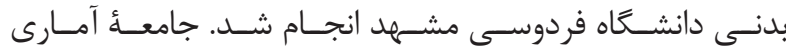

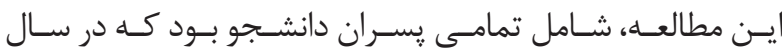

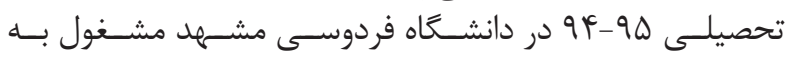

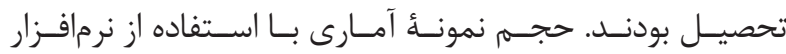

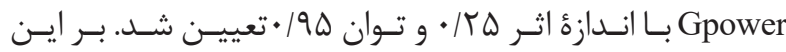

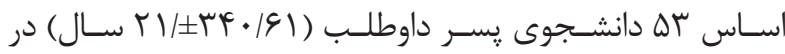

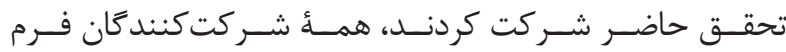

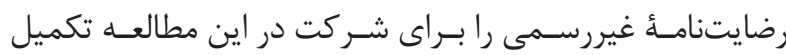

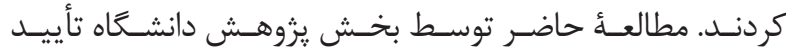

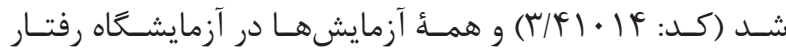

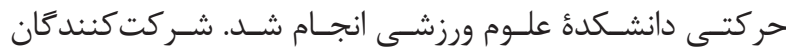

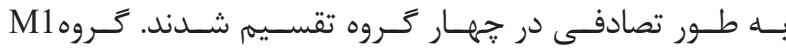
right
مقلممه

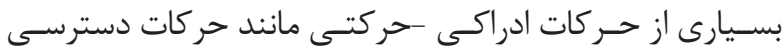

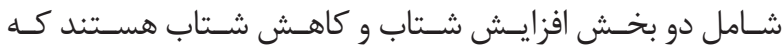

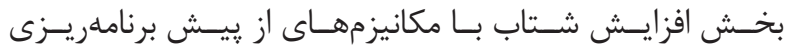

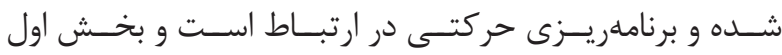

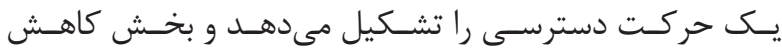

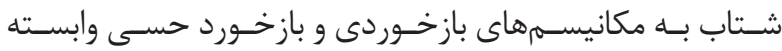

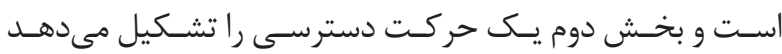

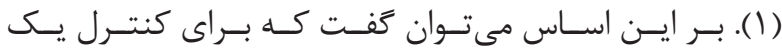

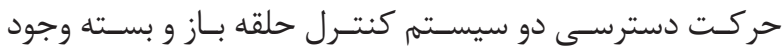

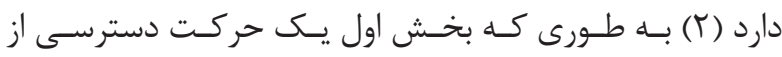

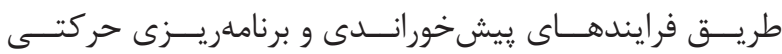

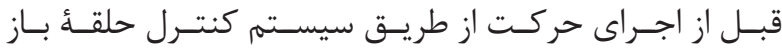

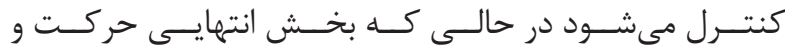

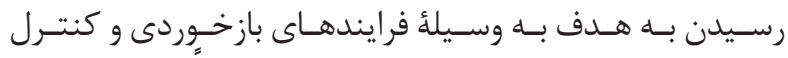

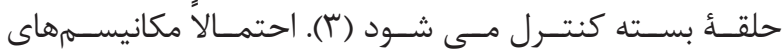

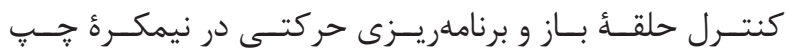

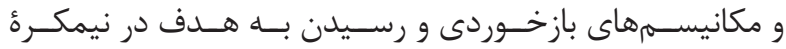

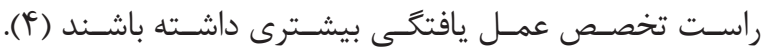

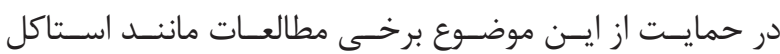

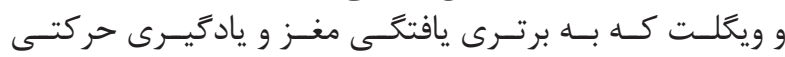

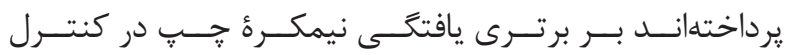

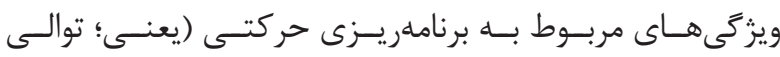

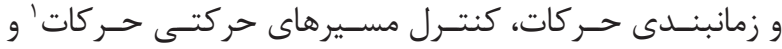

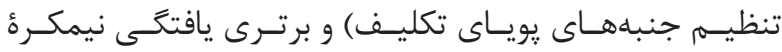

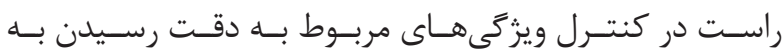

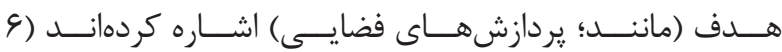

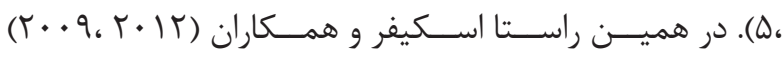

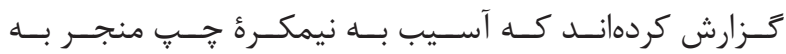

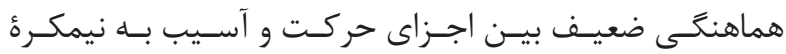

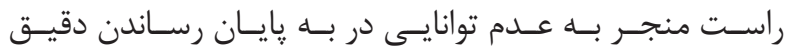

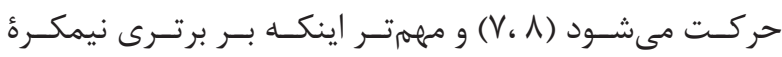

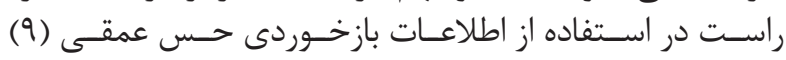

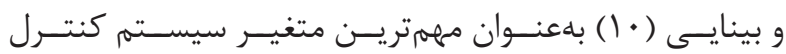

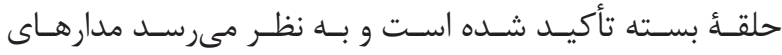

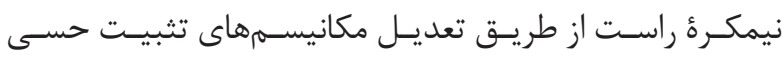

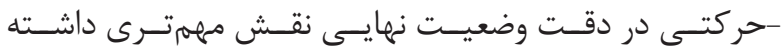

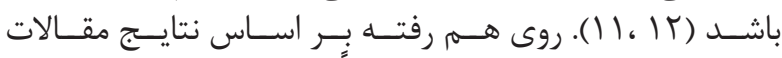

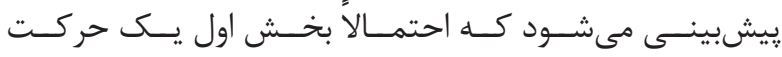

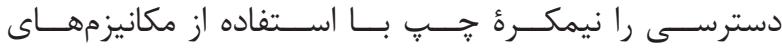

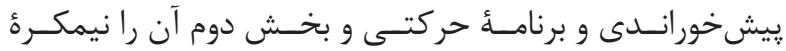

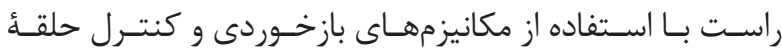

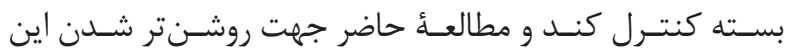

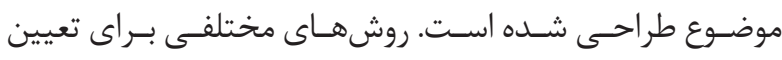

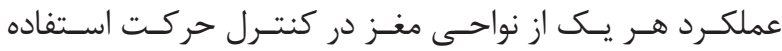

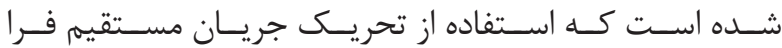

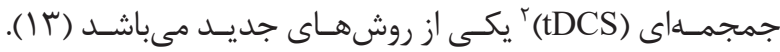




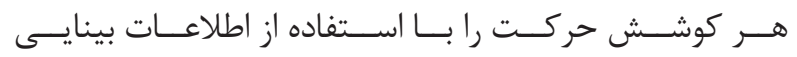

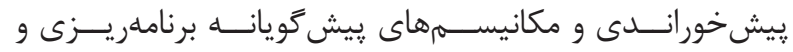

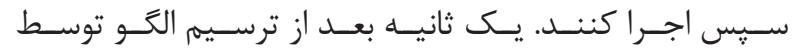

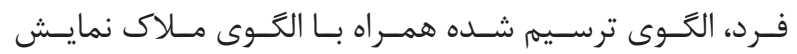

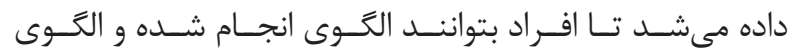

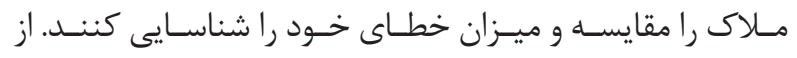

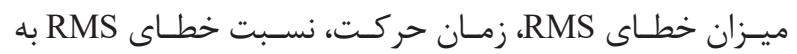

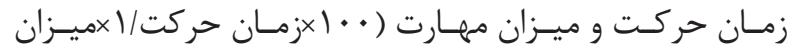

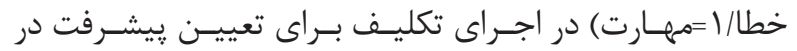

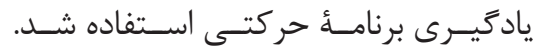

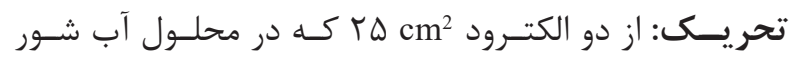

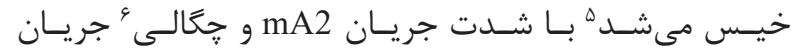

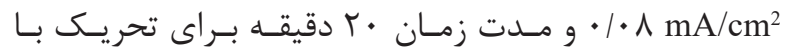

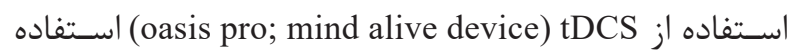

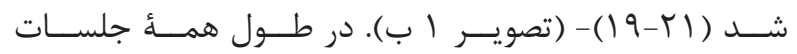

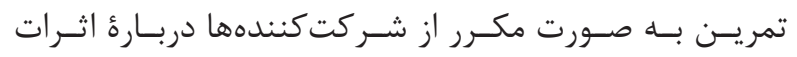

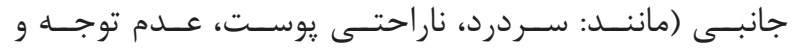

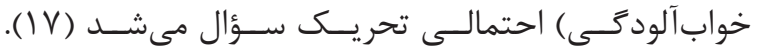

$$
\text { آزمايش دوم }
$$

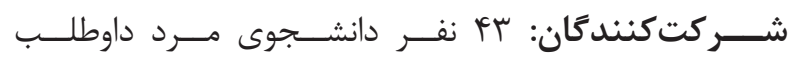

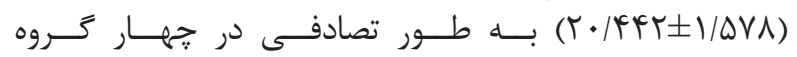
Sham tDCS $،(\mathrm{n}=1 /)$ DLPFC left، $(\mathrm{n}=\mid r)$ right ${ }^{~}$ DLPFC) (n=1) Practice ، (n=9)

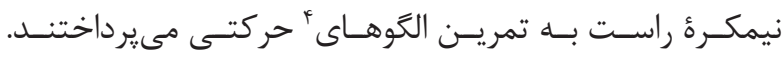

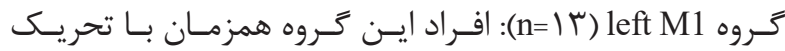

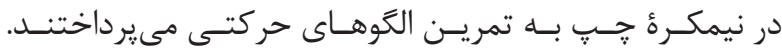

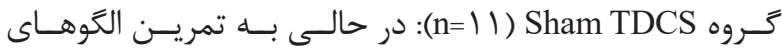

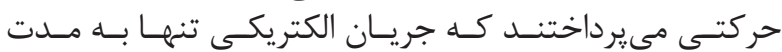

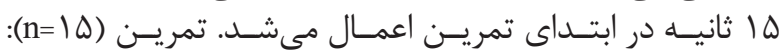

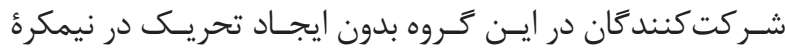

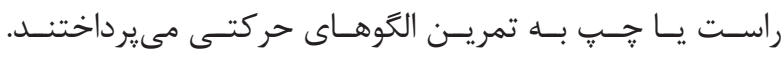

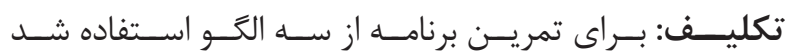

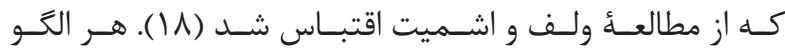

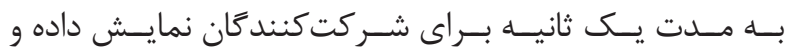

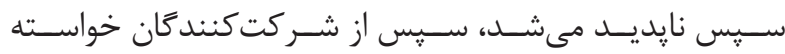

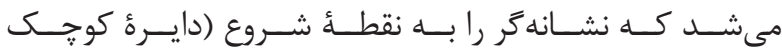

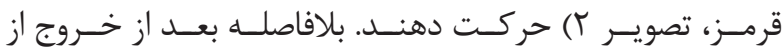

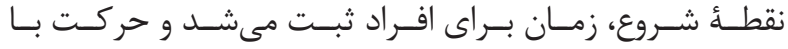

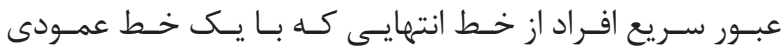

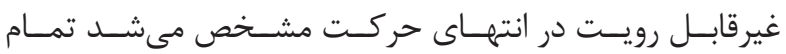

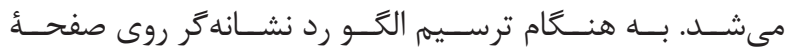

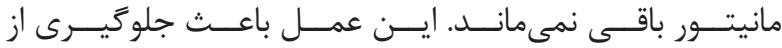

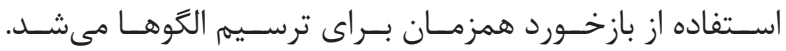

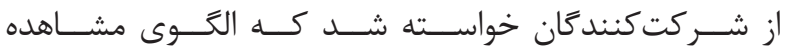

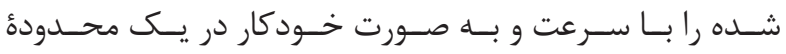

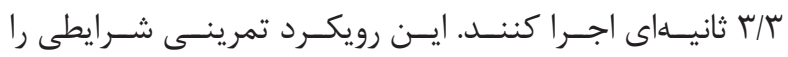

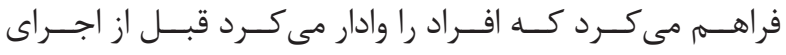

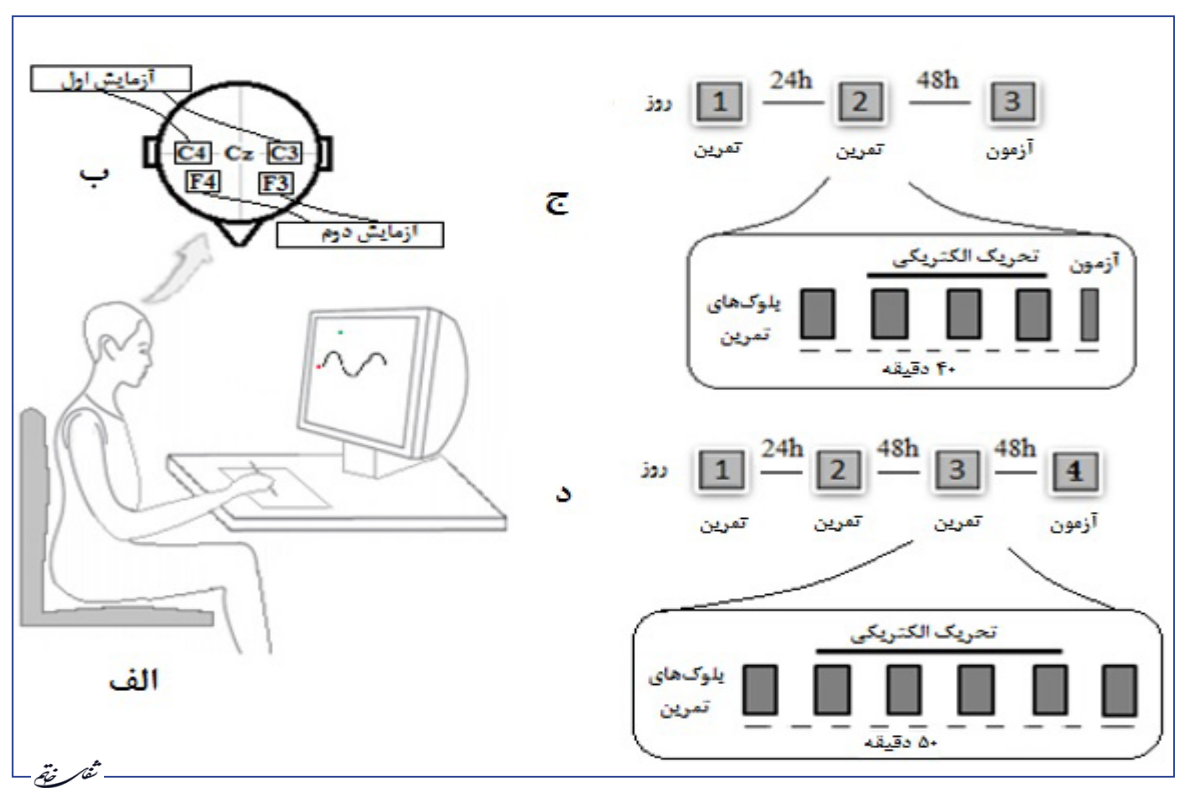

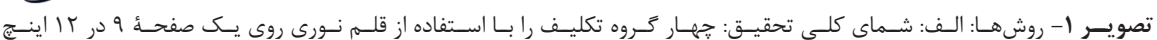

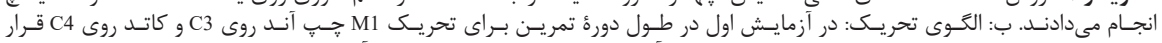

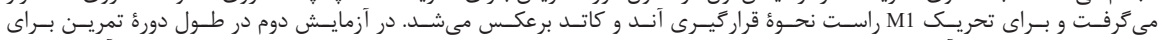

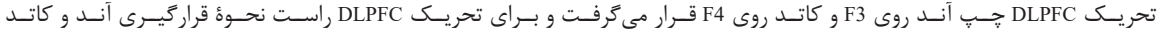

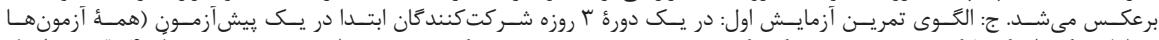

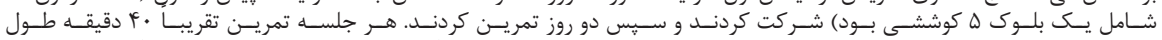

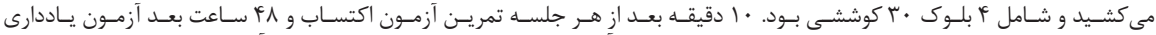

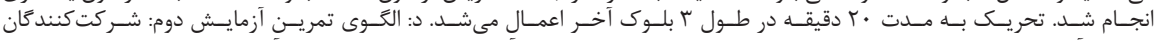

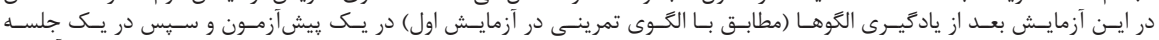

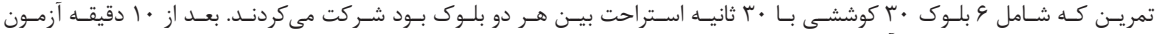

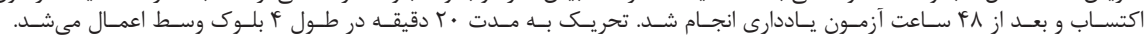

${ }^{4}$ Paradigm

${ }^{5}$ Soaked in a saline solution
${ }^{6}$ Density

${ }^{7}$ Dorsolateral prefrontal cortex 


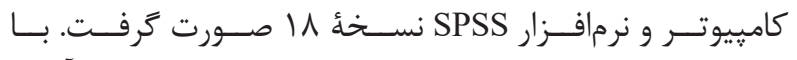

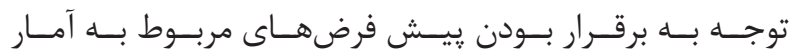

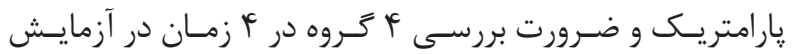

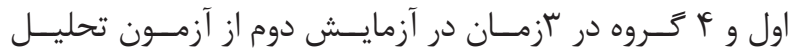

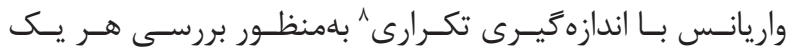

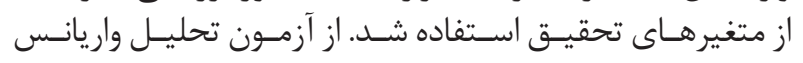

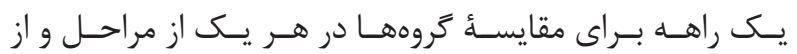

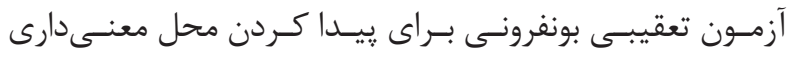

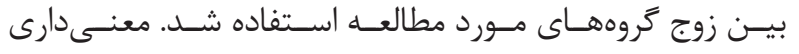

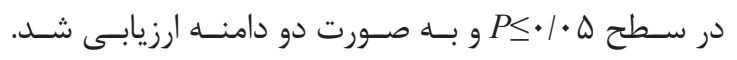

نافتهها

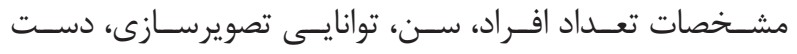

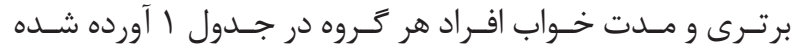

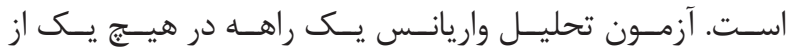

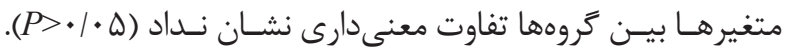

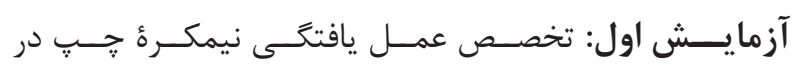

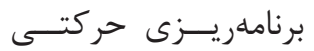

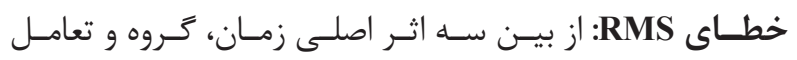

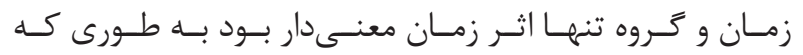

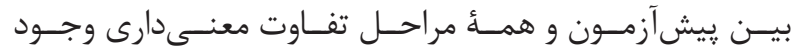

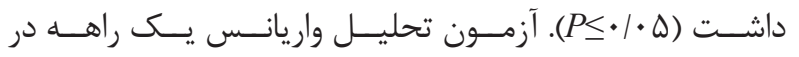

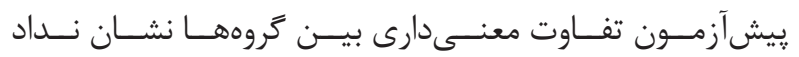

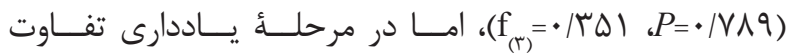

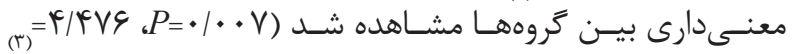

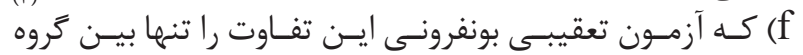
left M1

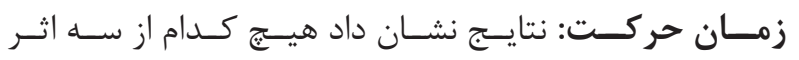

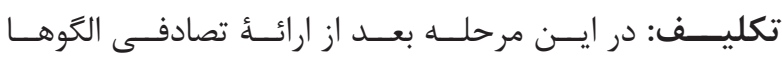

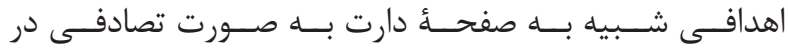

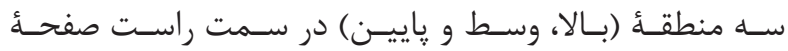

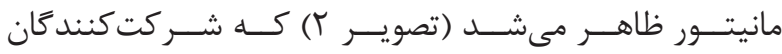

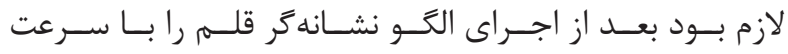

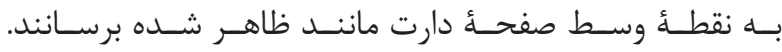

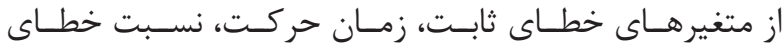

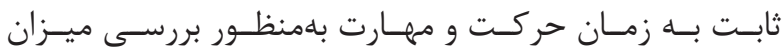

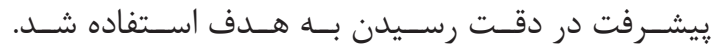

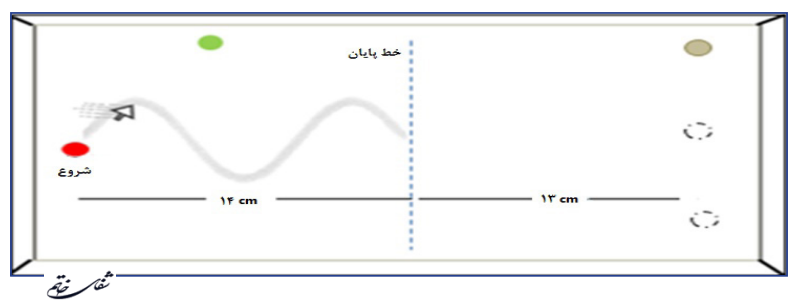

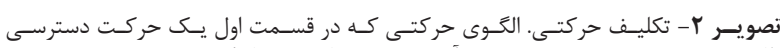

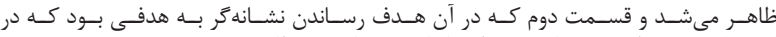

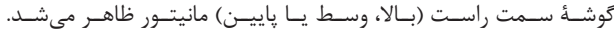

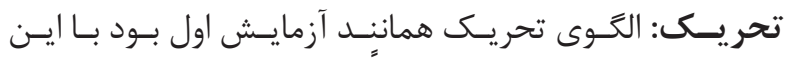

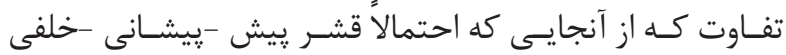

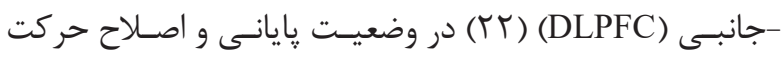

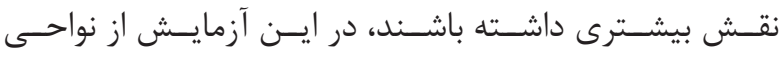

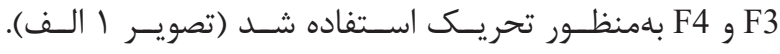

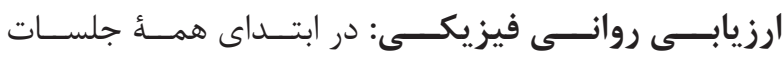

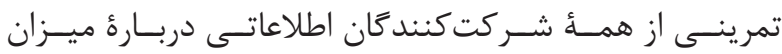

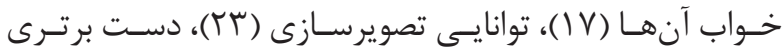

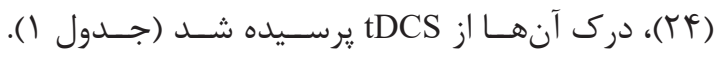

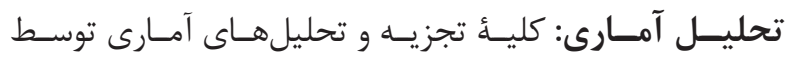

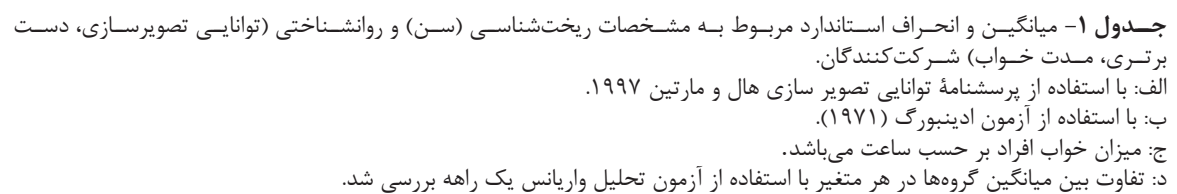

\begin{tabular}{|c|c|c|c|c|c|c|}
\hline مدت خواب (h)جه & دست برترى" & تواثايى تصويرسازى الف & سن (سال) & تعداد & ت كروه & \\
\hline$V / l f \pm 1 / \cdot r$ & $Q Q / F T \pm F / Q V$ & $f q / / f \pm g / V \lambda$ & $r \mid / 4 r \pm 1 / \Delta \Delta$ & if & Right M1 & \multirow{5}{*}{ آزمايش 1} \\
\hline$V / 9 \mid \pm 1 / I r$ & $৭ \Delta / r \wedge \pm \varphi / \varphi$. & $+\varepsilon / r \cdot \pm 9 / 9 \pi$ & $r \cdot / 9 q \pm 1 / q$. & ir & Left M1 & \\
\hline$V / r V \pm I / I$. & $99 / r f \pm 9 / V F$ & $f f / g r \pm V / V I$ & $r y / \cdot 9 \pm 1 / 44$ & $川$ & Sham tDCS & \\
\hline$v_{1} \cdot 9 \pm 1 / \cdot 9$ & $q f / g q \pm q /$ Tq & $f \Delta / r \xi \pm \xi / r \Lambda$ & $r T / \cdots \pm 1 / 9 q$ & 10 & Practice & \\
\hline$\cdot(\Delta V)$ & $\cdot / 1 \Delta S$ & . /ruf &.$/ 11$ & - & $P$ value ${ }^{2}$ & \\
\hline$V / \cdot 1 \pm 1 / T$. & $9 r / r r \pm \Delta 1 / 9$ & 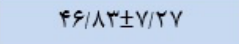 & $r \cdot / f 1 \pm 1 / \Delta$. & ir & Right DLPFC & \multirow{5}{*}{ آزمايش r r } \\
\hline$V / r q \pm 1 /+r$ & $9 f / \Delta f \pm g / \Lambda V$ & $F V / r q \pm 9 / 91$ & $r \cdot 1 \cdot 9 \pm 1 / r \cdot$ & 11 & Left DLPFC & \\
\hline $\mathrm{V} / \cdots \pm \cdot / 19$ & $Q V / V V \pm F / F$. & $\varphi g / \Delta \Delta \pm \varphi / \Delta r$ & $r \cdot / r T \pm r / 19$ & १ & Sham tDCS & \\
\hline$V / F \Delta \pm \cdot / 9 T$ & $9 \Delta / \uparrow \Delta \pm \xi / V \Lambda$ & $f F / \Lambda) \pm 9 / 1 \mathrm{~V}$ & $|r| / 1+ \pm|/ f|$ & 11 & Practice & \\
\hline$\cdot / 10$ & •/AT & | & $\cdot / \Delta 9$ & - & $P$ value ${ }^{2}$ & \\
\hline
\end{tabular}

\footnotetext{
${ }^{8}$ Repeated measure
} 


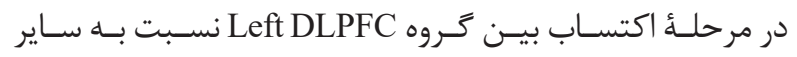

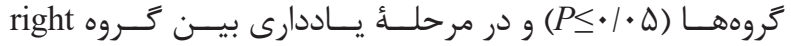

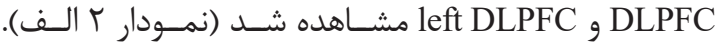

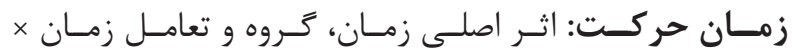

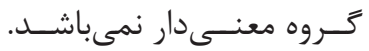

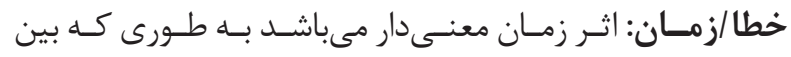

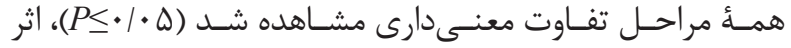

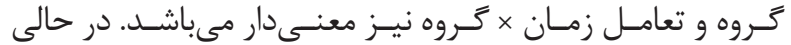

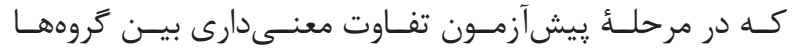

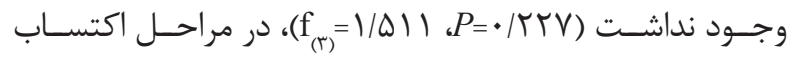

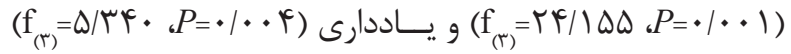

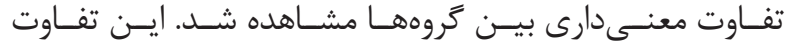

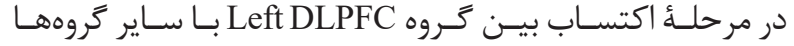

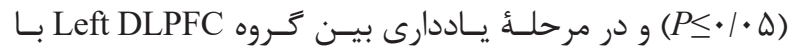

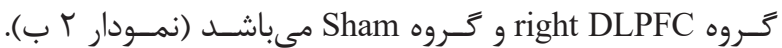
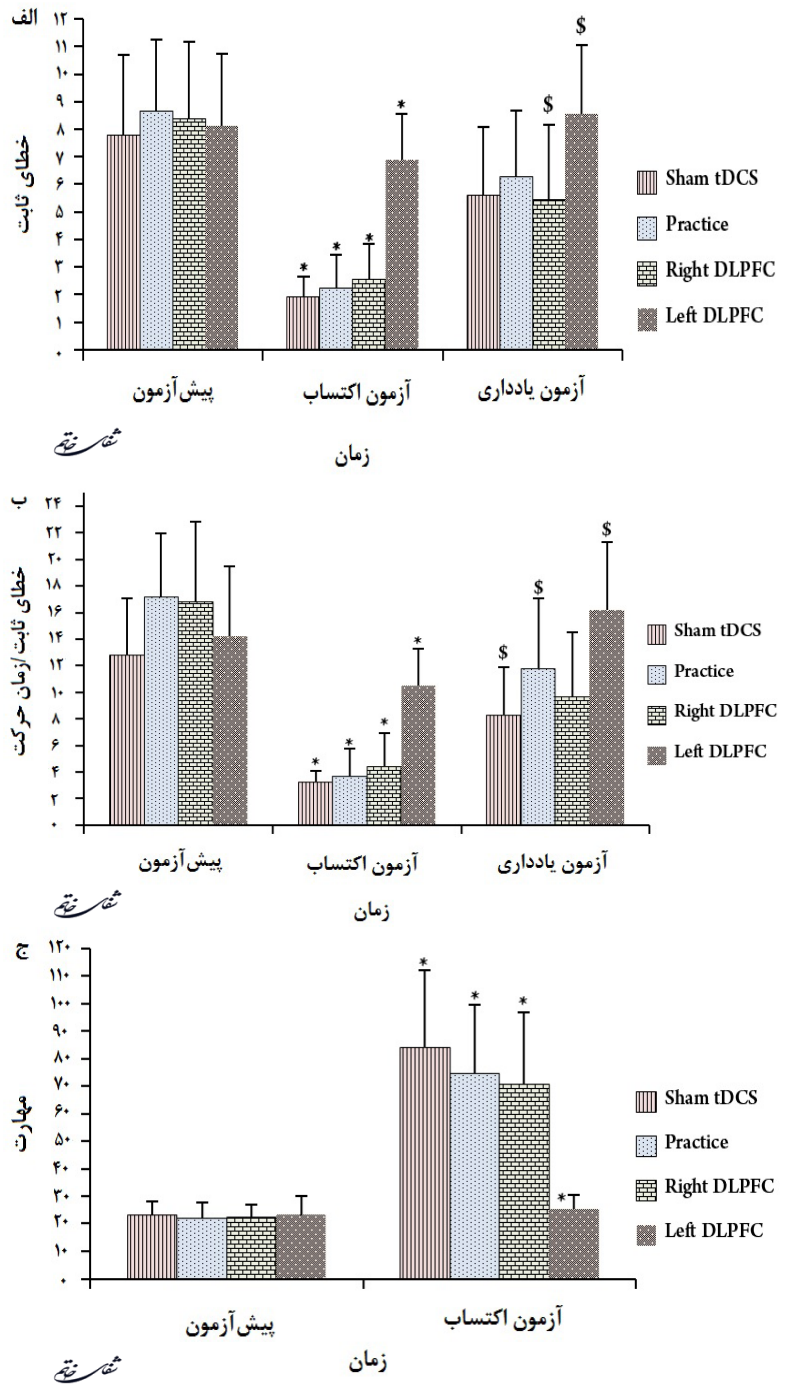

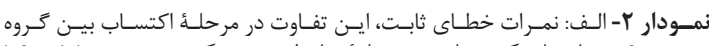

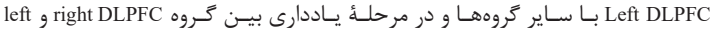

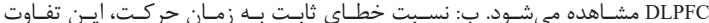

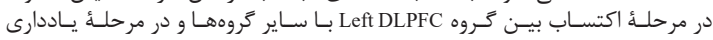

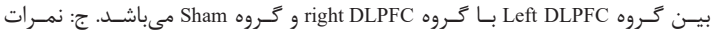

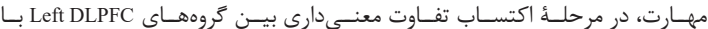

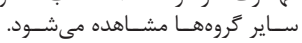

اصلى زمـان، گَروه و تعامـل زمـان و گَروه معنسى دار نيسـت.

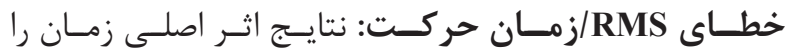

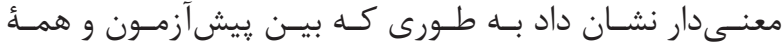

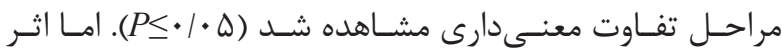

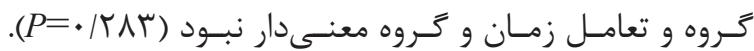

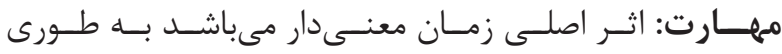

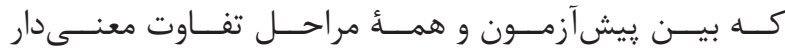

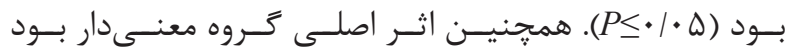
(P

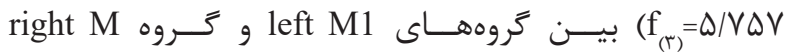

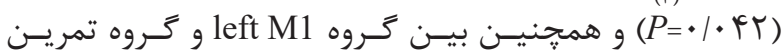

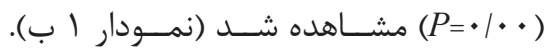
آزمايــش دوم: تخصـص عمـل يافتخَى نيمكــره راسـت در

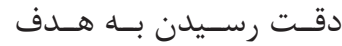
خطــاى ثابـت: اثـر اصلـى زمـان معنــى دار اسـت بــهـ طـورى

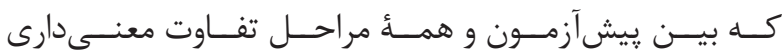

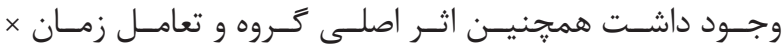

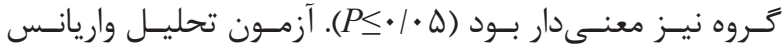

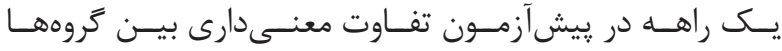
نشـان نـداد (rr/

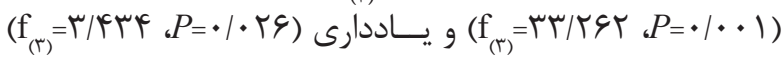

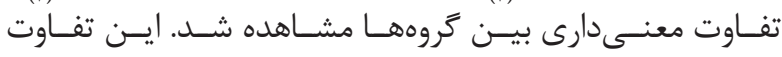
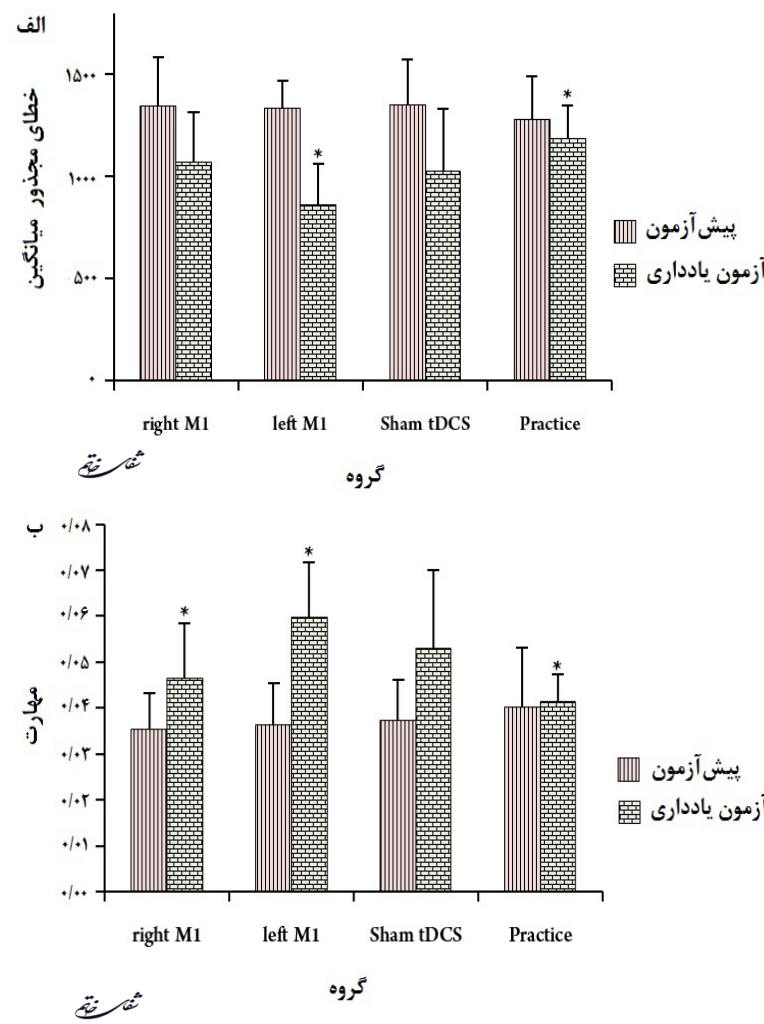

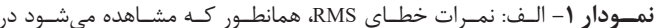

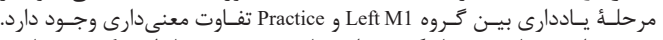

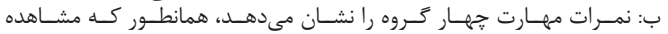

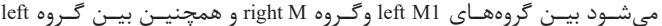

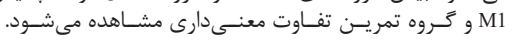




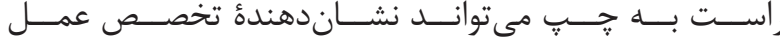

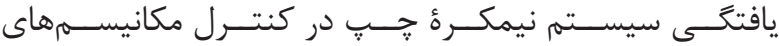

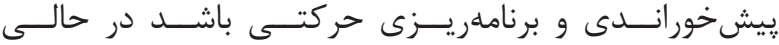

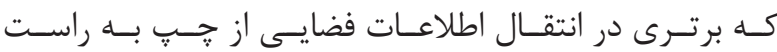

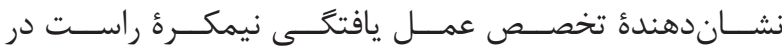

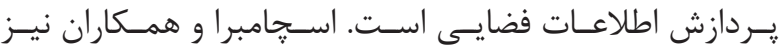

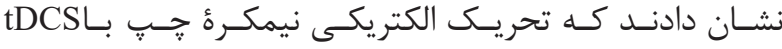

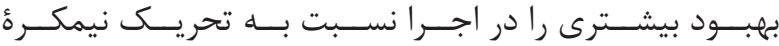

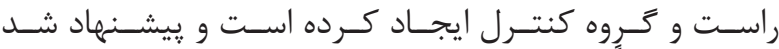

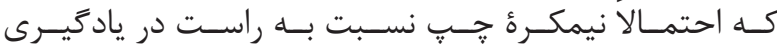

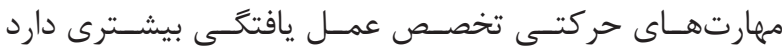

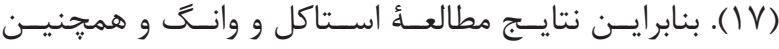

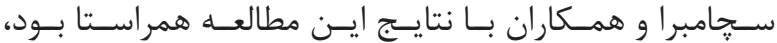

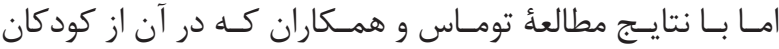

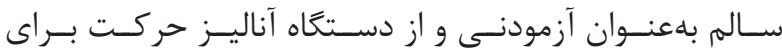

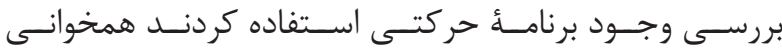

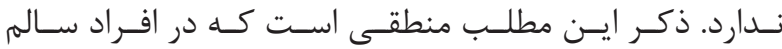

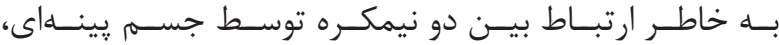

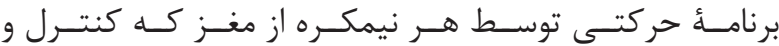

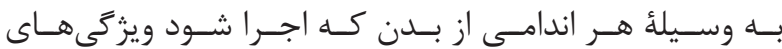

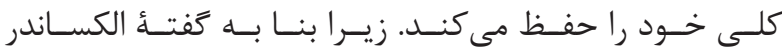

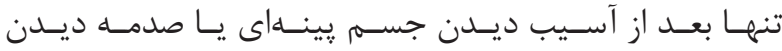

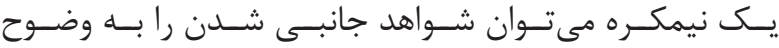

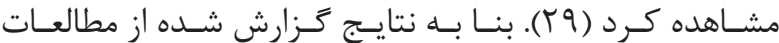

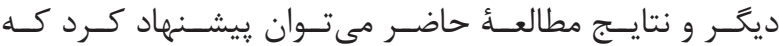

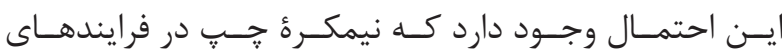

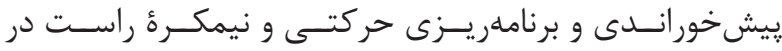

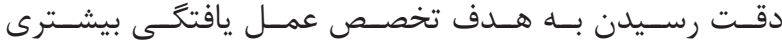
داشـته باشــند.

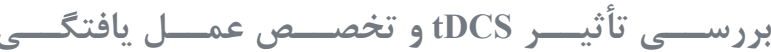

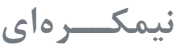

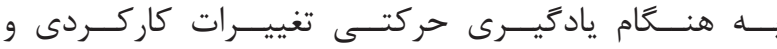

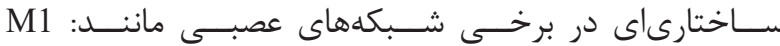

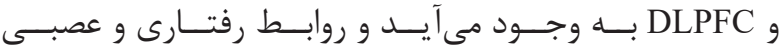

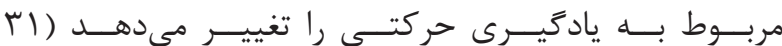

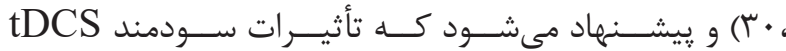

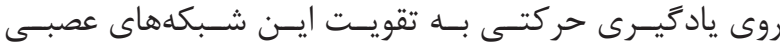

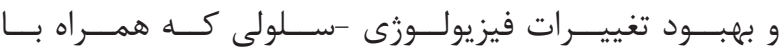

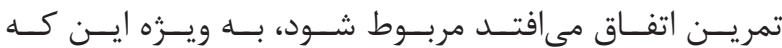

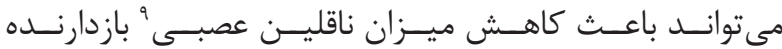

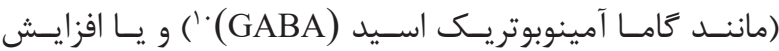

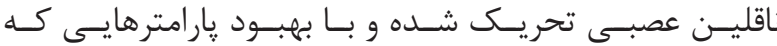

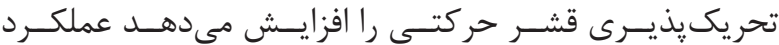

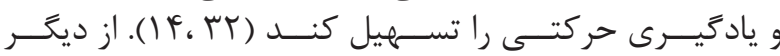

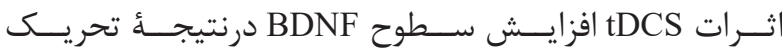

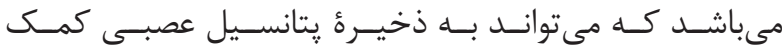

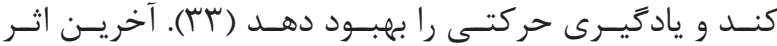

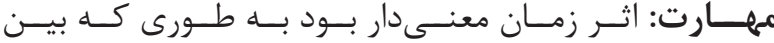

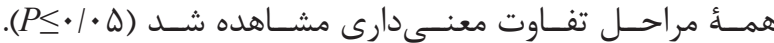

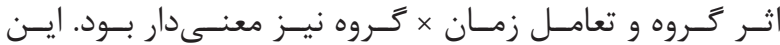

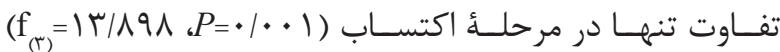

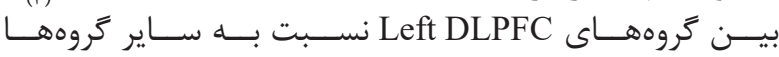

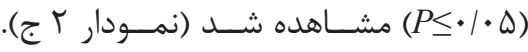

بحث و نتيجه كَيرى

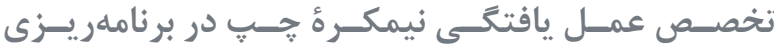
حر كت

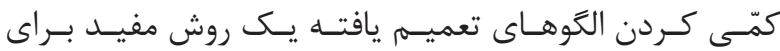

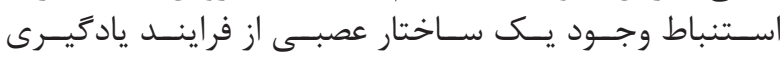

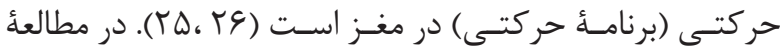

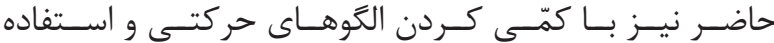

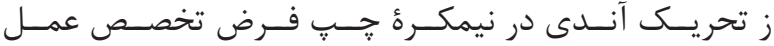

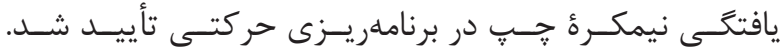

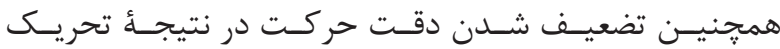

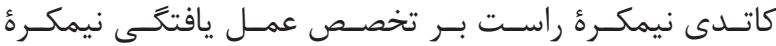

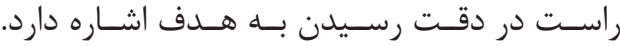

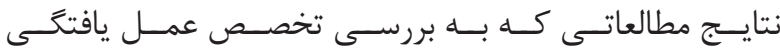

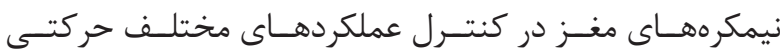

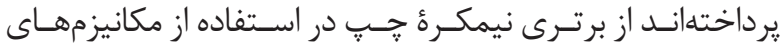

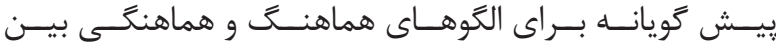

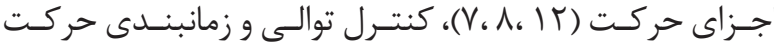

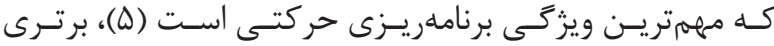

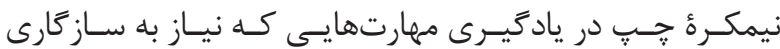

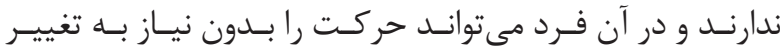

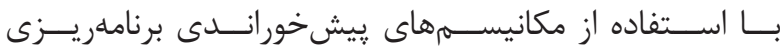

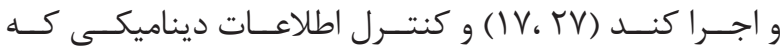

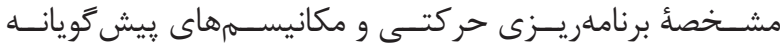

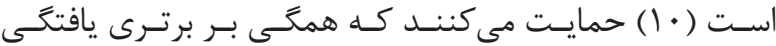

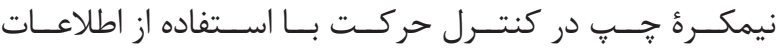

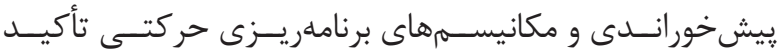

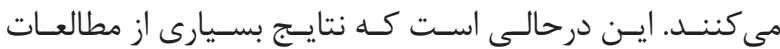

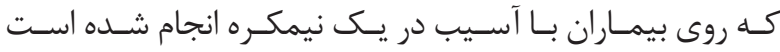

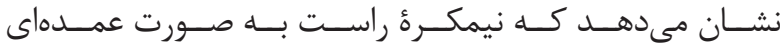

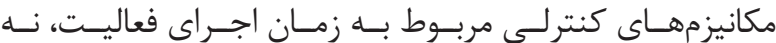

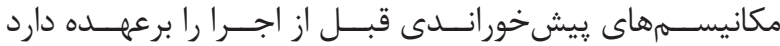

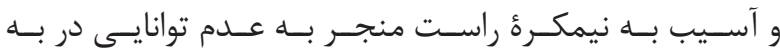

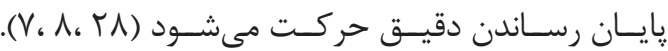

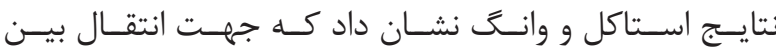

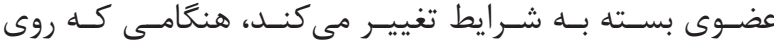

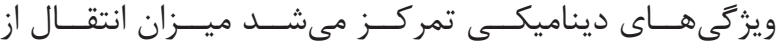

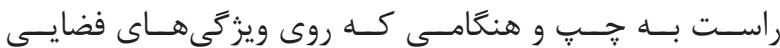

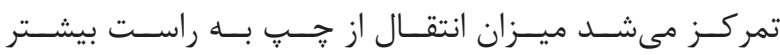

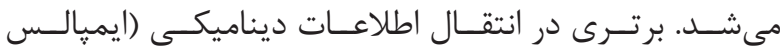

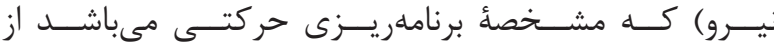

\footnotetext{
${ }^{9}$ Neurotransmitter

${ }^{10}$ gamma-aminobutyric acid
} 
تحريــ كاتـدى تأييـد شــد.

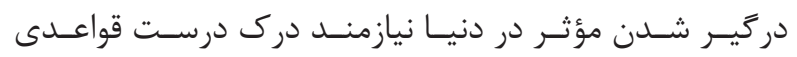

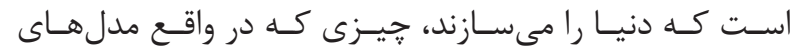

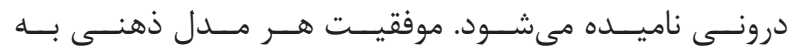

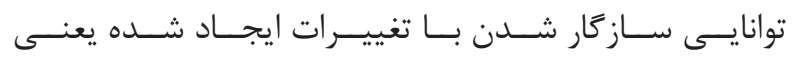

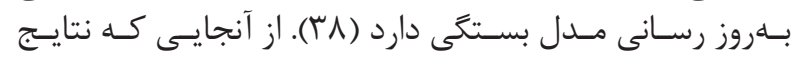

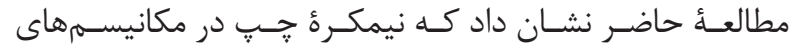

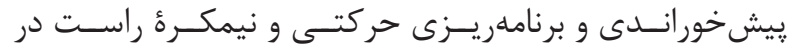

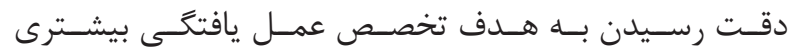

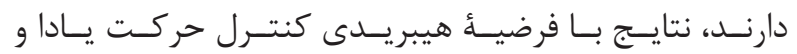

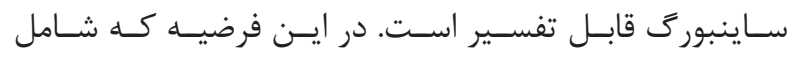

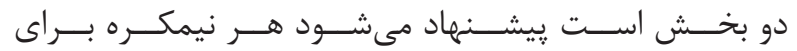

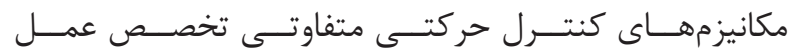

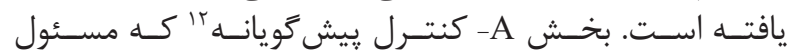

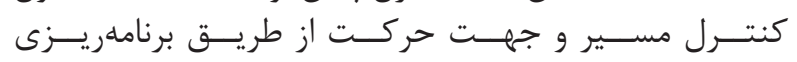

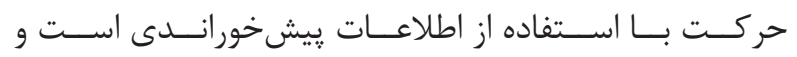

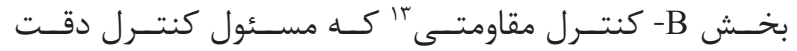

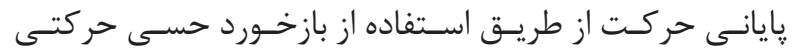

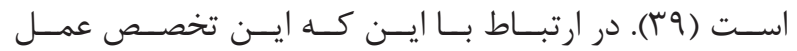

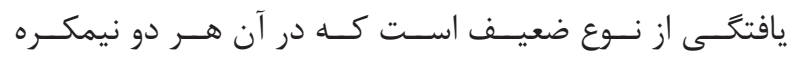

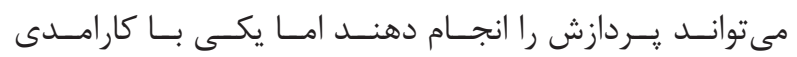

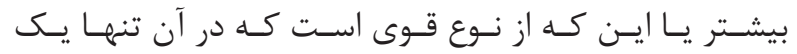

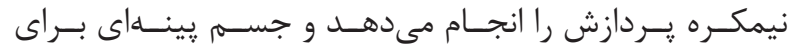

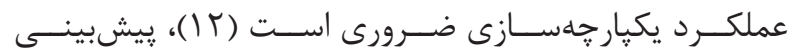

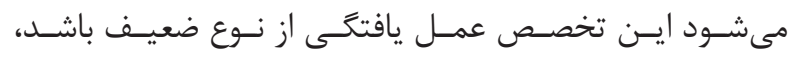

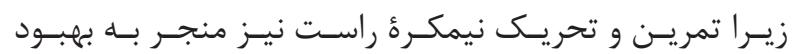

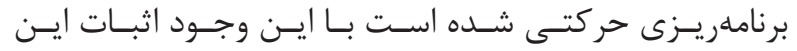

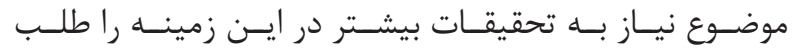

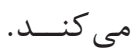

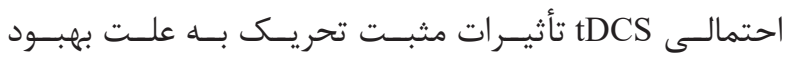

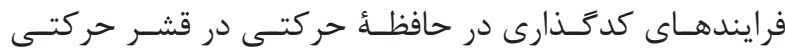

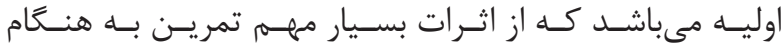

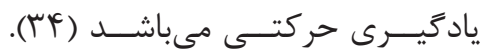

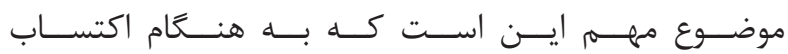

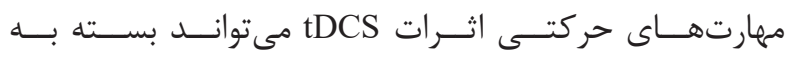

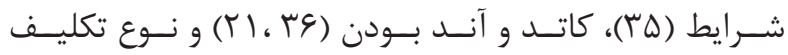

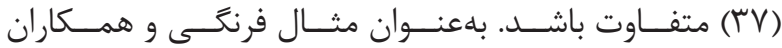

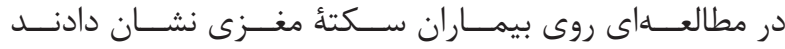

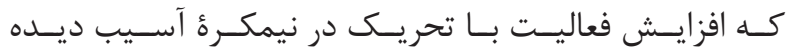

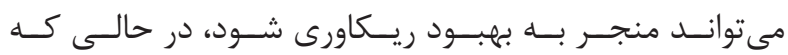

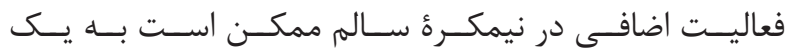

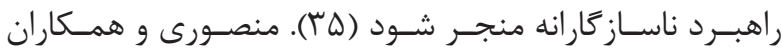

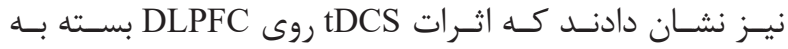

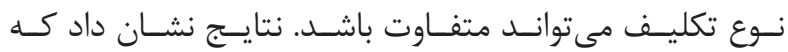

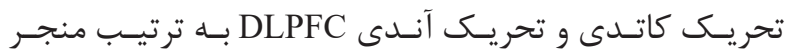

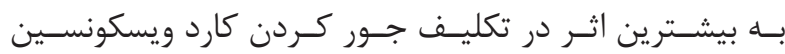
(WCST)

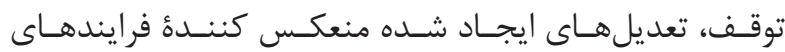

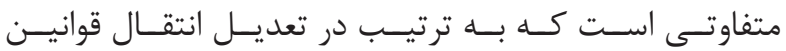

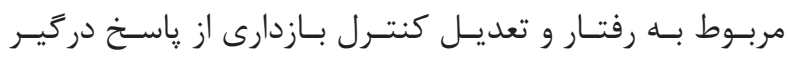

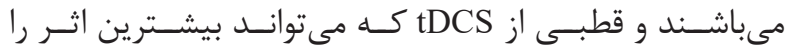

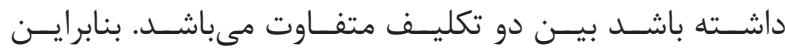

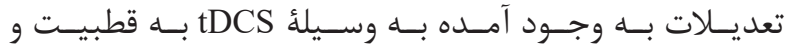

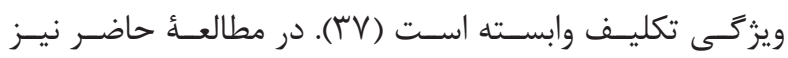

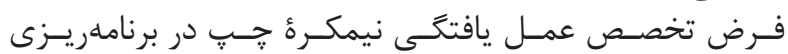

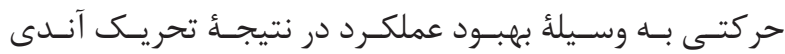

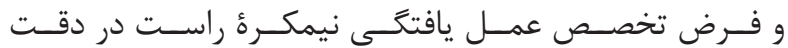

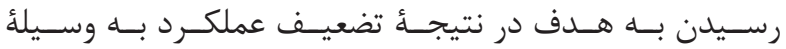

\footnotetext{
${ }^{11}$ Wisconsin card sorting test

${ }^{12}$ Predictive control

${ }^{13}$ Impedance control
} 
1. Sainburg RL, Schaefer SY. Interlimb differences in control of movement extent. J Neurophysiol. 2004; 92(3): 1374-83.

2. Serrien DJ, Ivry RB, Swinnen SP. Dynamics of hemispheric specialization and integration in the context of motor control. Nat Rev Neurosci. 2006; 7(2): 160-6.

3. Sainburg RL. Handedness: differential specializations for control of trajectory and position. Exerc Sport Sci Rev. 2005; 33(4): 206-13.

4. Sainburg RL. Evidence for a dynamic-dominance hypothesis of handedness. E Exp Brain Res. 2002; 142(2): 241-58.

5. Stöckel T, Weigelt M. Brain lateralisation and motor learning: selective effects of dominant and non-dominant hand practice on the early acquisition of throwing skills. Laterality. 2012; 17(1): 18-37.

6. Suzuki T, Higashi T, Takagi M, Sugawara K. Hemispheric asymmetry of ipsilateral motor cortex activation in motor skill learning. Neuroreport. 2013; 24(13): 693-7.

7. Schaefer SY, Haaland KY, Sainburg RL. Dissociation of initial trajectory and final position errors during visuomotor adaptation following unilateral stroke. Brain Research. 2009; 1298: 78-91.

8. Schaefer SY, Mutha PK, Haaland KY, Sainburg RL. Hemispheric specialization for movement control produces dissociable differences in online corrections after stroke. Cerebral Cortex. 2012; 22(6): 1407-19.

9. Goble DJ, Brown SH. Upper limb asymmetries in the matching of proprioceptive versus visual targets. Journal of Neurophysiology. 2008; 99(6): 3063-74.

10. Stöckel T, Wang J. Transfer of short-term motor learning across the lower limbs as a function of task conception and practice order. Brain and Cognition. 2011; 77(2): 271-9.

11. Mutha PK, Sainburg RL, Haaland KY. Critical neural substrates for correcting unexpected trajectory errors and learning from them. Brain. 2011; 134(12): 3647-61.

12. Mutha PK, Haaland KY, Sainburg RL. The effects of brain lateralization on motor control and adaptation. Journal of Motor Behavior. 2012; 44(6): 455-69.

13. Marquez CMS, Zhang X, Swinnen SP, Meesen R, Wenderoth N. Task-specific effect of transcranial direct current stimulation on motor learning. Front Hum Neurosci. 2013; 7: 333. doi: 10.3389/fnhum.2013.00333.
14. Reis J, Fritsch B. Modulation of motor performance and motor learning by transcranial direct current stimulation. Current Opinion in Neurology. 2011; 24(6): 590-6.

15. Hunter T, Sacco P, Nitsche MA, Turner DL. Modulation of internal model formation during force field-induced motor learning by anodal transcranial direct current stimulation of primary motor cortex. The Journal of Physiology. 2009; 587(12): 2949-61.

16. Nitsche MA, Schauenburg A, Lang N, Liebetanz D, Exner C, Paulus W, et al. Facilitation of implicit motor learning by weak transcranial direct current stimulation of the primary motor cortex in the human. Journal of Cognitive Neuroscience. 2003; 15(4): 619-26.

17. Schambra HM, Abe M, Luckenbaugh DA, Reis J, Krakauer JW, Cohen LG. Probing for hemispheric specialization for motor skill learning: a transcranial direct current stimulation study. Journal of Neurophysiology. 2011; 106(2): 652-61.

18. Wulf G, Schmidt RA. Feedback-induced variability and the learning of generalized motor programs. Journal of Motor Behavior. 1994; 26(4): 348-61.

19. Stagg C, O'shea J, Kincses Z, Woolrich M, Matthews P, Johansen-Berg H. Modulation of movementassociated cortical activation by transcranial direct current stimulation. European Journal of Neuroscience. 2009; 30(7): 1412-23.

20. Wagner T, Fregni F, Fecteau S, Grodzinsky A, Zahn M, Pascual-Leone A. Transcranial direct current stimulation: a computer-based human model study. Neuroimage. 2007; 35(3): 1113-24.

21. Lindenberg R, Renga V, Zhu L, Nair D, Schlaug G. Bihemispheric brain stimulation facilitates motor recovery in chronic stroke patients. Neurology. 2010; 75(24): 2176-84.

22. Shadmehr R, Holcomb HH. Neural correlates of motor memory consolidation. Science. 1997; 277(5327): 821-5.

23. Hall CR, Martin KA. Measuring movement imagery abilities: a revision of the movement imagery questionnaire. Journal of Mental Imagery. 1997; 21(1-2): 143-54.

24. Oldfield RC. The assessment and analysis of handedness: the edinburgh inventory. Neuropsychologia. 1971; 9(1): 113-97.

25. Shadmehr R. Generalization as a behavioral window 
to the neural mechanisms of learning internal models. Human Movement Science. 2004; 23(5): 543-68.

26. de Xivry J-JO, Marko MK, Pekny SE, Pastor D, Izawa J, Celnik P, et al. Stimulation of the human motor cortex alters generalization patterns of motor learning. The Journal of Neuroscience. 2011; 31(19): 7102-10.

27. Stöttinger E, Filipowicz A, Marandi E, Quehl N, Danckert J, Anderson B. Statistical and perceptual updating: correlated impairments in right brain injury. Experimental Brain Research. 2014; 232(6): 1971-87.

28. Mutha PK, Sainburg RL, Haaland KY. Left parietal regions are critical for adaptive visuomotor control. The Journal of Neuroscience. 2011; 31(19): 6972-81.

29. Kalat JW. Biological Psychology. $9^{\text {th }}$ ed. 2007; p. 287.

30. Ungerleider LG, Doyon J, Karni A. Imaging brain plasticity during motor skill learning. Neurobiol Learn Mem. 2002; 78(3): 553-64.

31. De Xivry J-jO, Shadmehr R. Electrifying the motor engram: effects of tDCS on motor learning and control. Exp Brain Res. 2014; 232(11): 3379-95.

32. Reis J, Robertson EM, Krakauer JW, Rothwell J, Marshall L, Gerloff C, et al. Consensus: can transcranial direct current stimulation and transcranial magnetic stimulation enhance motor learning and memory formation? Brain Stimul. 2008; 1(4): 363-9.
33. Fritsch B, Reis J, Martinowich K, Schambra HM, Ji Y, Cohen LG, et al. Direct current stimulation promotes BDNF-dependent synaptic plasticity: potential implications for motor learning. Neuron. 2010; 66(2): 198-204.

34. Bütefisch CM, Khurana V, Kopylev L, Cohen LG. Enhancing encoding of a motor memory in the primary motor cortex by cortical stimulation. Journal of Neurophysiology. 2004; 91(5): 2110-6.

35. Fregni F, Boggio PS, Mansur CG, Wagner $T$, Ferreira MJ, Lima MC, et al. Transcranial direct current stimulation of the unaffected hemisphere in stroke patients. Neuroreport. 2005; 16(14): 1551-5.

36. Vines BW, Nair D, Schlaug G. Modulating activity in the motor cortex affects performance for the two hands differently depending upon which hemisphere is stimulated. European Journal of Neuroscience. 2008; 28(8): 1667-73.

37. Mansouri FA, Fehring DJ, Feizpour A, Gaillard A, Rosa M, Rajan R, et al. Direct current stimulation of prefrontal cortex modulates error-induced behavioral adjustments. Eur J Neurosci. 2016; 44(2): 1856-69.

38. Danckert J, Anderson B. Updating representations of temporal intervals. Experimental Brain Research. 2015; 233(12): 3517-26.

39. Yadav V, Sainburg RL. Handedness can be explained by a serial hybrid control scheme. Neuroscience. 2014; 278:385-96. 